\title{
Rôle anti-arythmogène de l'endothéline dans l'ischémie cardiaque
}

L'endothéline (ET) est une hormone peptidique sécrétée constitutivement par les cellules endothéliales des vaisseaux sanguins et de l'endocarde. Il existe trois isopeptides codés par trois gènes différents. Ces endothélines, ET-1, ET-2 et ET-3, activent deux récepteurs à sept passages transmembranaires, $\mathrm{ET}_{\mathrm{A}}$ et $\mathrm{ET}_{\mathrm{B}}$, qui appartiennent à la superfamille des récepteurs couplés aux protéines $G$ [1]. Les activités biologiques des endothélines sont nombreuses et incluent les effets vasoconstricteurs ainsi que des effets mitogènes sur diverses cellules dont celles du muscle vasculaire lisse [1-3]. En dépit de ces effets bien documentés, le rôle physiologique ou physiopathologique des endothélines demeure mal défini. L'importance physiologique de l'effet mitogène de l'ET-1 a été mise en évidence récemment chez la souris après inactivation par recombinaison homologue du gène de l'endothéline-1 $\left(\mathrm{m} / \mathrm{s} n^{\circ} 6-7\right.$, vol. $\left.10, p .740\right)$ [3]. Les souris homozygotes ET-1-/développaient de nombreuses anomalies craniofaciales et mouraient à la naissance d'insuffisance respiratoire. En revanche, les souris hétérozygotes $E T-1+/-$, qui produisaient moitié moins d'ET-1 que les souris normales, étaient viables mais hypertendues, un phénotype inattendu considérant l'effet vasoconstricteur important de l'ET-1. Néanmoins, ce phénotype est consistant avec un rôle de l'ET-1 dans l'homéostasie cardiovasculaire. Par ailleurs, plusieurs observations suggèrent un rôle de l'ET1 dans la physiopathologie cardiovasculaire. Par exemple, l'ET-1 est induite dans plusieurs conditions de stress, telles que l'insuffisance cardiaque et l'infarctus du myocarde. Cependant, le rôle de cette synthèse et le mécanisme d'action de l'ET-1 étaient mal définis. Deux récentes $m / s n^{\circ} 2$, vol. 11, favrier 95 publications dans la revue Nature [5, 6] proposent que l'ET-1 jouerait un rôle modulateur des paramètres électrophysiologiques qui s'opposerait, lors de l'ischémie, à l'eff et arythmogène des catécholamines. Dans l'article de James et al. (Ciba Geigy Japon, université d'Okazaki et Kyoto, Japon) [5], l'électrophysiologie des cardiomyocytes ventri-culaires de cobayes fut étudiée par la méthode du patch-clamp. Dans ces cellules, les courants transmembranaires induits par un agoniste adrénergique, l'isoprénaline, et provoqués par l'activation de canaux chlorure dépendants de la protéine kinase $\mathrm{A}$, sont inhibés de façon dépendante de la dose par ET-1. L'inhibition par l'endothéline-1 peut être abolie par une augmentation de la concentration de l'isoprénaline, suggérant un mécanisme compétitif entre l'isoprénaline et l'endothéline pour l'activation des courants $\mathrm{Cl}^{-}$. Cette inhibition reflète le mécanisme sous-jacent à l'activation des canaux chlorure dépendants de la PKA où l'adénylyl cyclase est soumise aux effets opposés des protéines $G_{s}$ et $G_{i}$. L'inhibition du courant $\mathrm{Cl}^{-}$induit par les catécholamines est abolie par la toxine de Pertussis (PTX) et l'endothéline-1 diminue l'élévation du courant provoquée par l'histamine et la forskoline, deux agents inducteurs de l'AMPc. De plus, l'endothéline-1 diminue significativement la concentration de base de l'AMPc. Cette inhibition est complètement abolie par l'antagoniste sélectif du récepteur $\mathrm{ET}_{\mathrm{A}} \mathrm{BQ}-123$, mais pas par l'antagoniste sélectif d'ET $\mathrm{d}_{\mathrm{B}}$, sarfatoxine $\mathrm{S} 6 \mathrm{c}$ (SRTXc), suggérant que certains effets de l'ET-1 sont sélectivement relayés par le récepteur $\mathrm{ET}_{\mathrm{A}}$. Ono et al. (Tokyo et Kyoto, Japon), quant à eux, ont utilisé des myocytes dispersés d'oreillettes de lapin pour étudier le rôle de l'endothéline-1 [6]. Leurs résultats montrent que l'ET-1, via le récepteur $\mathrm{ET}_{\mathrm{A}}$, inhibe le canal potassique par le même mécanisme, c'està-dire via l'inhibition de l'adénylyl cyclase et la réduction de la concentration intracellulaire de l'AMPc.

L'endothéline-1 jouerait donc, dans les conditions physiopathologiques où elle est relarguée avec les catécholamines, un effet paracrine et cardioprotecteur, en modulant l'électrophysiologie du myocarde. Cependant, avant de conclure définitivement sur un rôle anti-arythmogène de l'endothéline-1, son effet devra être testé in vivo dans des conditions basales ainsi que dans des conditions d'ischémie. Dans ce contexte, le rôle anti-arythmogène de l'ET-1 pourrait expliquer le phénotype hypertendu des souris ET-1+/- ; la diminution de l'ET-1 étant équivalente à une stimulation $\beta$-adrénergique continue conduirait à une augmentation du rythme cardiaque; par ailleurs, la réponse de ces animaux aux arythmies et à l'infarctus du myocarde serait bien intéressante à explorer, considérant qu'ils représentent un modèle génétique de déficience de l'endothéline-1.

D.D., M.N.

1. Lotersztajn S. Les endothélines. médecine/ sciences $1993 ; 9$ : 1084-93.

2. Luscher TF, Oemar BS, Boulanger CM, Hahn AW. Molecular and cellular biology of endothelin and its receptors. Part I.J Hypertens $1993 ; 11$ : 7-11. 3. Luscher TF, Oemar BS, Boulanger CM, Hahn AW. Molecular and cellular biology of endothelin and its receptors. Part II. J Hypertens $1993 ; 11$ 121-6.

4. Kurihara Y, Kurihara H, Suzuki H, et al. Elevated blood pressure and craniof acial abnormalities in mice deficient in endothelin-1. Nature 1994; $368: 703-10$

5. James AF, Xie LH, Fujitani Y, Hayashi S, Horie $\mathrm{M}$. Inhibition of the cardiac protein kinase A-dependent chloride conductance by endothelin-1. Nalure 1994; 370 : 297-300.

6. Ono K, Tsujimoto G, Sakamoto A, Eto K, Masaki T, Ozaki Y, Satake M. Endothelin-A receptor mediates cardiac inhibition by regulating calcium and potassium currents. Nature 1994, 370 : 301-4.

(n)

De

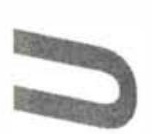

